\title{
Robótica Educacional e Pensamento Computacional: uma Avaliação da Percepção dos Alunos sobre o Tema
}

\author{
Ana Beatriz T. B. Dornelles1, Cristhyan A. Cruz2, Elizabet M. S. Medeiros3, \\ João Victor A. Araújo1, Kely D. V. Villacorta3, Lorena C. L. Buritiı \\ 1Graduando em Ciência da Computação \\ 2 Graduando em Matemática Computacional \\ 3Departamento de Computação Científica \\ Centro de Informática-Universidade Federal da Paraíba (UFPB) \\ João Pessoa - PB - Brasil \\ biadornelles.2011@gmail.com, cristhyan.cruz@mat.ci.ufpb.br, \\ elizabet@ci.ufpb.br, johnalcoforado@gmail.com, kelydvveci.ufpb.br, \\ lorenaburitiecc.ci.ufpb.br
}

Resumo. O ensino da Robótica Educacional tem se mostrado desafiador principalmente no quesito da compreensão dos conceitos da programação. As técnicas de Computação Desplugada aliadas ao Pensamento Computacional tem se mostrado um meio eficaz para auxiliar os alunos de Escolas Estaduais na utilização, implementação, montagem e programação dos Kits de Robótica Educacional. Este trabalho mostra alguns métodos utilizados para trabalhar a resolução de problemas, a projeção de sistemas e vários outros conceitos da ciência da computação, e a percepção dos alunos do $1^{\circ}$ Ano do Ensino Médio após 4 meses de implantação do projeto.

Abstract. Teaching Educational Robotics is a challenging mainly because of programming concepts. The techniques of Unplugged Computing allied to Computational Thinking have proven to be an effective means to assist State School students in the use, implementation, assembly and programming of Educational Robotics Kits. This work shows some methods used to work with problem solving, systems projection and several other concepts of computer science, and the students' perception of the 1st year of high school after 4 months of project implementation.

\section{Introdução}

O projeto Robótica Educacional e Pensamento Computacional é um projeto de extensão (Edital PROBEX 2019) com vigência de 10 meses e início em março de 2019. Esse projeto visa auxiliar os alunos e professores na utilização, implementação, montagem e programação dos Kits de Robótica, recebidos através do Projeto de Robótica Educacional do Governo da Paraíba. O uso do pensamento computacional foi incorporado para facilitar a resolução de problemas, projeção de sistemas e compreensão do comportamento humano, através da extração de conceitos fundamentais da ciência da computação [Wing 2006]. Esse projeto está sendo realizado com os alunos do $1^{\circ}$ ano do ensino médio da Escola Cidadã Integral Mons. Pedro Anísio Bezerra Dantas, em João Pessoa-PB. 
O uso de uma metodologia própria de ensino, lúdica e ao mesmo tempo didática, baseada nos conceitos de computação desplugada [Bell 2011] e pensamento computacional [Oliveira 2016], mostrou-se eficaz para o desenvolvimento das competências necessárias à robótica educacional. Procura-se fazer com que os alunos pensem de forma crítica, lógica e sistemática para resolução de problemas [Bordini 2017]. Além disso, a montagem dos kits de robótica permite aos alunos o desenvolvimento cognitivo, a experiência do trabalho em equipe e da divisão de tarefas [Oliveira 2016].

Todas as atividades propostas foram desenvolvidas visando os 4 pilares do pensamento computacional [Wing 2006]: Decomposição-onde os alunos tinham que identificar um problema complexo e quebrá-lo em pedaços menores e mais fáceis de gerenciar; Reconhecimento de padrões- através da análise individual dos problemas menores com maior profundidade, se pode identificar problemas parecidos, que já foram solucionados anteriormente; Abstração- foco apenas nos detalhes que são importantes, enquanto informações irrelevantes são ignoradas; Algoritmo- Passos ou regras simples que podem ser criados para resolver cada um dos subproblemas encontrados.

Foram desenvolvidos diversos materiais didáticos para às aulas de pensamento computacional e utilizados os kits de robótica da Fischertechnik (2019) disponíveis na escola. Também foram incorporadas aulas com o software de programação visual em blocos, Code.org, para estabelecer a relação entre o pensamento computacional e a programação dos robôs.

A ideia principal é fazer como sugere Blikstein (2008), introduzir a tecnologia como um Cavalo de Tróia, usando ferramentas familiares, práticas e atividades lúdicas, mediadas por professores, com potencial de causar mudança afetiva e conceitual. De forma que, "quando os estudantes apropriam-se da tecnologia de Tróia ... são capazes de realizar investigações e construções autênticas" [Blikstein 2008]. Repassando de forma sutil os conceitos da Ciência da Computação [Bundy 2007].

\section{Materiais e Métodos}

O Projeto PROBEX é realizado por uma equipe de 2 alunos e 2 alunas coordenados por 2 professoras do Centro de Informática da UFPB. O planejamento das atividades é realizado em reuniões semanais e leva em consideração os resultados obtidos nas atividades anteriores. A escola disponibilizou dois laboratórios para execução do PROBEX, um de Robótica (Figura 1) e outro de Informática (Figura 2).

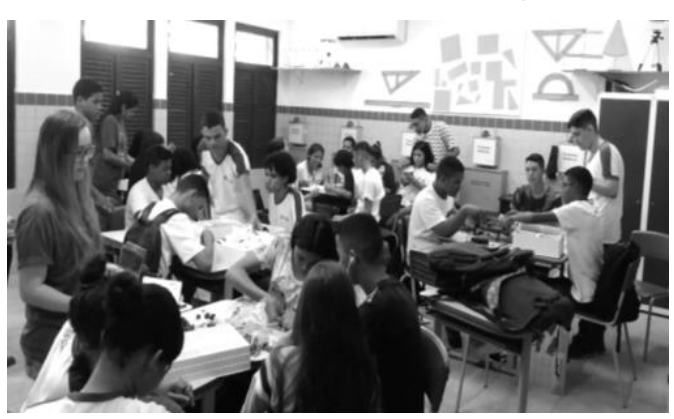

Figura 1. Laboratório de Robótica

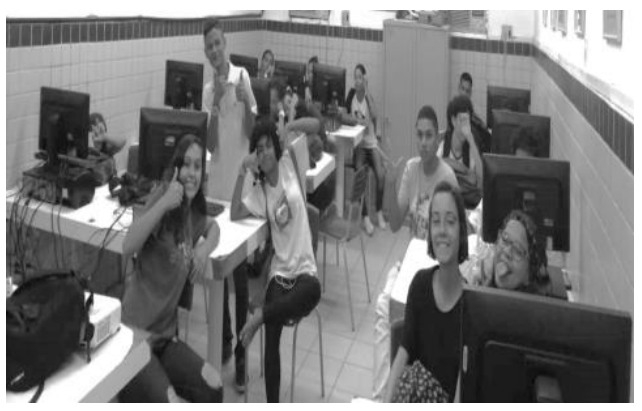

Figura 2. Laboratório de Informática

A metodologia aplicada nas aulas foi: mediante o uso de dinâmicas, trazer de forma lúdica os conceitos de pensamento computacional e de programação de robôs. 
Desta forma foram trabalhados, por exemplo, conceitos de criptografia (Figura 3) e de algoritmo de busca binária (Figura 4).

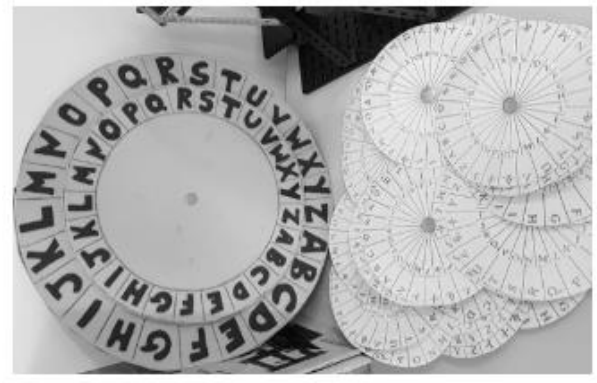

Figura 3. Cifra de Cesar

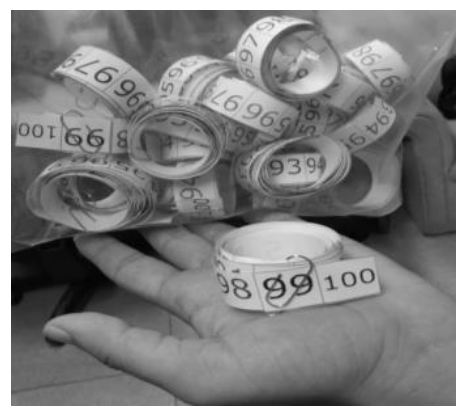

Figura 4. Fita métrica

A programação foi exercitada por intermédio do site Code.org (2015), aonde os alunos utilizaram determinados jogos para exercitar e fixar o uso da lógica de programação na solução dos problemas apresentados. Desta forma, foram trabalhados os conceitos de programação em blocos, condicionais e laços de repetição.

Segundo Zilli (2002) o ensino da robótica nas escolas tem como objetivo

desenvolver o raciocínio lógico do aluno, facilitar o uso de conceitos aprendidos em outras áreas de conhecimento para desenvolver projetos, trabalhar conceitos de desenho, física, álgebra e geometria, estimular a utilização de teorias em atividades concretas [Zilli 2002].

Os indicadores deste projeto foram baseados nos resultados de dois questionários elaborados pela equipe do PROBEX sobre o tema do projeto, usando perguntas diretas, de múltipla escolha e de fácil entendimento. Os questionários foram aplicados no início do projeto e após quatro meses de projeto.

\section{Resultados}

Tabela 1. Comparativo entre dados do Questionário Inicial e do Questionário aplicado após 4 meses de Projeto, com relação ao GÊNERO dos estudantes

\begin{tabular}{|l|c|c|}
\hline Participantes Gênero & $\begin{array}{c}\text { Questionário Inicial } \\
\text { Freq. }\end{array}$ & $\begin{array}{c}\text { Quest. Após quatro meses de projeto. } \\
\text { Freq. }\end{array}$ \\
\hline Masculino & $49 \%$ & $45 \%$ \\
\hline Feminino & $49 \%$ & $55 \%$ \\
\hline Outro & $2 \%$ & $0 \%$ \\
\hline
\end{tabular}

Tabela 2. Comparativo das respostas dos estudantes sobre o nível de dificuldade em resolver problemas

\begin{tabular}{|c|c|c|c|c|c|}
\hline \multicolumn{3}{|c|}{ Questionário Inicial } & \multicolumn{3}{c|}{ Questionário após quatro meses de projeto } \\
\hline Perguntas & Respostas & Freq. & Perguntas & Respostas & Freq. \\
\hline
\end{tabular}




\begin{tabular}{|c|c|c|c|c|c|}
\hline \multirow{3}{*}{$\begin{array}{l}\text { Sente dificuldade } \\
\text { em resolver } \\
\text { problemas de } \\
\text { lógica }\end{array}$} & Sempre / Quase sempre & $38 \%$ & \multirow{3}{*}{$\begin{array}{c}\text { Consegue } \\
\text { visualizar melhor } \\
\text { a resolução de } \\
\text { problemas de } \\
\text { lógica }\end{array}$} & Nunca / Quase Nunca & $14 \%$ \\
\hline & Na maioria das vezes & $41 \%$ & & Na maioria das vezes & $36 \%$ \\
\hline & Nunca / Quase Nunca & $21 \%$ & & Sempre / Quase Sempre & $50 \%$ \\
\hline \multirow{3}{*}{$\begin{array}{l}\text { Sente dificuldade } \\
\text { em resolver } \\
\text { problemas de } \\
\text { matemática }\end{array}$} & Sempre / Quase sempre & $46 \%$ & \multirow{3}{*}{$\begin{array}{l}\text { Consegue } \\
\text { perceber como } \\
\text { aplicar, na } \\
\text { prática, os } \\
\text { conteúdos vistos } \\
\text { durante o projeto }\end{array}$} & Nunca / Quase Nunca & $14 \%$ \\
\hline & Na maioria das vezes & $24 \%$ & & Na maioria das vezes & $41 \%$ \\
\hline & $\begin{array}{l}\text { Nunca / Quase Nunca } \\
\text { /Não respondeu }\end{array}$ & $30 \%$ & & Sempre / Quase Sempre & $46 \%$ \\
\hline
\end{tabular}

Tabela 3. Comparativo sobre a percepção dos estudantes, sobre robótica educacional e pensamento computacional

\begin{tabular}{|l|l|c|c|}
\hline \multicolumn{1}{|c|}{ Perguntas } & \multicolumn{1}{c|}{ Respostas } & $\begin{array}{c}\text { Questionário Inicial } \\
\text { Freq. }\end{array}$ & $\begin{array}{c}\text { Quest. após quatro meses } \\
\text { de projeto - Freq. }\end{array}$ \\
\hline \multirow{2}{*}{$\begin{array}{l}\text { Você sabe o que é } \\
\text { Robótica } \\
\text { educacional? }\end{array}$} & Claro que sei / Sei & $16 \%$ & $91 \%$ \\
\cline { 2 - 4 } & Acho que sei & $24 \%$ & $9 \%$ \\
\cline { 2 - 4 } & Não sei & $59 \%$ & $0 \%$ \\
\hline $\begin{array}{l}\text { Você já sabe o que é } \\
\text { um Algoritmo? }\end{array}$ & Claro que sei / Sei & $14 \%$ & $68 \%$ \\
\cline { 2 - 4 } & Acho que sei & $16 \%$ & $18 \%$ \\
\cline { 2 - 4 } & Não sei / Não respondeu & $71 \%$ & $14 \%$ \\
\hline \multirow{2}{*}{$\begin{array}{l}\text { Você já sabe o que é } \\
\text { Pensamento } \\
\text { Computacional? }\end{array}$} & Claro que sei / Sei & $5 \%$ & $45 \%$ \\
\cline { 2 - 4 } & Acho que sei & $38 \%$ & $41 \%$ \\
\cline { 2 - 5 } & Não sei / Não respondeu & $57 \%$ & $14 \%$ \\
\hline
\end{tabular}

Tabela 4. Comparativo sobre o interesse inicial dos estudantes em participar do projeto e a motivação em permanecer no projeto após 4 meses

\begin{tabular}{|c|c|c|c|c|}
\hline Questionário Inicial & Respostas & Freq. & $\begin{array}{c}\text { Quest. após } 4 \text { meses } \\
\text { de Projeto }\end{array}$ & Freq. \\
\hline \multirow{3}{*}{$\begin{array}{l}\text { Interesse em participar do } \\
\text { projeto de robótica } \\
\text { educacional e pensamento } \\
\text { computacional. }\end{array}$} & Muito alto / Alto & $25 \%$ & \multirow{3}{*}{$\begin{array}{l}\text { Motivação em } \\
\text { permanecer no } \\
\text { projeto }\end{array}$} & $68 \%$ \\
\hline & Moderado & $57 \%$ & & $18 \%$ \\
\hline & Baixo & $19 \%$ & & $14 \%$ \\
\hline
\end{tabular}


Tabela 5. Comparativo sobre o interesse dos estudantes nas atividades realizadas durante o projeto, aplicado após quatro meses de projeto

\begin{tabular}{|l|c|c|c|}
\hline \multicolumn{1}{|c|}{ Interesse nas Atividades realizadas } & $\begin{array}{c}\text { Muito alta / Alta } \\
\text { Freq. }\end{array}$ & Moderada Freq. & Baixa Freq. \\
\hline Utilização do Code.Org & $73 \%$ & $23 \%$ & $14 \%$ \\
\hline Utilização dos Kits de Robótica & $77 \%$ & $18 \%$ & $5 \%$ \\
\hline Aula temática & $81 \%$ & $14 \%$ & $5 \%$ \\
\hline Aula teórica & $59 \%$ & $41 \%$ & $0 \%$ \\
\hline
\end{tabular}

\section{Discussão}

O total de alunos que responderam ao Questionário Inicial foi de 37 estudantes e o Questionário aplicado após 4 meses de projeto obteve 22 respostas. A faixa etária dos entrevistados variou de 14 a 15 anos, em 2 turmas do $1^{\circ}$ ano do ensino médio. A Tabela 1 mostra a declaração de gênero dos entrevistados e revela que houve um aumento no interesse dos estudantes do gênero feminino de $45 \%$ para $55 \%$ após quatro meses de atividades do projeto.

A robótica educacional é uma área multidisciplinar que integra disciplinas como: matemática, engenharia mecânica, inteligência artificial, inglês, história, entre outras. É um instrumento que permite ao professor demonstrar na prática conteúdos abordados teoricamente, tornando o ensino motivador ao aluno e melhorando sua capacidade de aprendizado [Zilli 2004]. A introdução de tecnologias como método didático pode colaborar com o aumento da procura pelo conhecimento e o desenvolvimento de muitas outras habilidades necessárias ao aluno.

Os alunos entrevistados foram indagados sobre o nível de dificuldades em resolver problemas e foi possível observar os resultados na Tabela 2, onde 38\% sempre ou quase sempre relatam dificuldade em resolver problemas de lógica e que $46 \%$ sempre ou quase sempre sente dificuldade em resolver problemas de matemática. Após quatro meses de atividades do projeto pôde-se observar que estes números já melhoraram pois agora apenas $14 \%$ ainda não consegue visualizar como resolver problemas de lógica e nem perceber como aplicar, na prática, os conteúdos vistos durante o projeto. Foi perceptível também observar que antes $21 \%$ não sentia dificuldades com lógica e, após 4 meses de projeto, $50 \%$ afirma que consegue visualizar como resolver problemas de lógica sempre ou quase sempre. O importante foi verificar que $46 \%$ (sempre ou quase sempre) e que $41 \%$ (na maioria das vezes) dos entrevistados afirmam que conseguem perceber como aplicar, na prática, os conteúdos vistos durante o projeto.

A Tabela 3 apresenta a percepção dos alunos no início e após 4 meses, com relação aos conhecimentos base do projeto. É possível verificar que no início apenas $16 \%$ afirmava saber o que é robótica educacional, agora são $91 \%$. O pensamento computacional foi assimilado por $45 \%$, contra 5\% no início do projeto. O conceito de algoritmo que no inicio $71 \%$ afirmava que não sabia ou não respondeu foi assimilado completamente por $68 \%$ dos estudantes.

$\mathrm{O}$ interesse dos estudantes em participar do projeto e a motivação em permanecer no projeto após 4 meses pode ser visto na Tabela 4. Demonstrando que 
antes de saber do que se tratava o projeto somente $25 \%$ dos estudantes tinham interesse, mas depois de quatro meses participando efetivamente do projeto, $68 \%$ afirma interesse e motivação em permanecer participando.

Foi verificado também qual o tipo de atividade havia sido mais interessante do ponto de vista de aprendizado para os estudantes. A Tabela 5 apresenta a seguinte classificação com relação ao interesse dos estudantes: Aula temática com $81 \%$, Utilização dos Kits de Robótica com 77\%, Utilização do Code.Org com 73\% e as Aulas Teóricas com 59\%.

\section{Conclusão}

Os resultados, embora parciais, pois ainda faltam 5 meses para o fim do Projeto, já indicam pontos de avanço significativos como a compreensão dos conceitos básicos do projeto, vistos na Tabela 3, pensamento computacional, robótica educacional e algoritmos não são mais palavras incompreensíveis.

A mudança na relação dos alunos com o projeto, (Tabela 4), onde 68\% demonstra interesse e motivação para continuar participando, foi também observada pela equipe do projeto durante as aulas, a cada aula os alunos demonstravam cada vez mais interesse e evolução tanto no raciocínio logico quanto no pensamento computacional, realizando as atividades cada vez mais rapidamente. E isso também pôde ser verificado pelos resultados da Tabela 2 , onde $50 \%$ sempre ou quase sempre consegue visualizar melhor a resolução de problemas de lógica e $36 \%$ consegue na maioria das vezes.

Foi possível identificar as atividades que mais prenderam a atenção dos alunos (Tabela 5), classificando-as e permitindo à equipe confirmar que atividades mais lúdicas, com temas que relacionam a teoria à prática, são a melhor forma de repassar o conhecimento de pensamento computacional e robótica educacional.

Finalmente notou-se que, na percepção dos alunos, os assuntos deste projeto são refletidos em outras matérias, de tal forma que eles estão utilizando os conhecimentos aprendidos para agregar em outras disciplinas. Conforme pode ser visto na Tabela 2, onde $46 \%$ sempre ou quase sempre consegue perceber como aplicar, na prática, os conteúdos vistos durante o projeto e $41 \%$ consegue na maioria das vezes.

\section{Referências}

Bell, T.; Witten, I. H.; Fellows (2011) M. "Computer Science Unplugged - Ensinando Ciência da Computação sem o uso do computador", Disponível em: https://classic.csunplugged.org/wp-content/uploads/2014/12/CSUnpluggedTeachersportuguese-brazil-feb-2011.pdf, Acesso em: 04 de maio de 2017.

Blikstein, P. in Noguera, P. \& Silva, C. A.(2008) "Freire and the Possible Dream", TRAVELS IN TROY WITH FREIRE - Technology as an Agent of Emancipation, Sense Publishers, Rotterdam, Disponível em: http://www.blikstein.com/paulo/ documents/books/Blikstein-TravelsInTroyWithFreire.pdf, Acesso em: 15 de junho de 2019.

Bordini, Adriana et al. (2017) "Pensamento Computacional nos Ensinos Fundamental e Médio: uma revisão sistemática", In: Brazilian Symposium on Computers in Education (Simpósio Brasileiro de Informática na Educação-SBIE), p. 123. 
Brackmann, Christian et al. (2017) "Pensamento Computacional Desplugado: Ensino e Avaliação na Educação Primária Espanhola", In: Anais dos Workshops do Congresso Brasileiro de Informática na Educação, p. 982.

Bundy A. (2007) "Computational Thinking Is Pervasive", Journal of Scientific and Practical Computing, vol. 1, n. 2, pp. 67-69.

Code.org (2013) What is Code.org? [S.1.], Disponível em: http://Code.org, Acesso em: 10 de novembro de 2016.

FischerTechnik (2019) Disponível em: http://www.fischerbrasil.com.br. Acesso em: 15 de junho de 2019.

Oliveira, E. J. S. de. (2016), "Pensamento computacional e robótica: um estudo sobre habilidades desenvolvidas em oficinas de robótica educacional". Monografia. UFPB. Disponível em: http://rei.biblioteca.ufpb.br:8080/jspui/bitstream/123456789/2857/1/ EJSO21062016.pdf, Acesso em: 15 de junho de 2019.

Wing, J. M. (2006). “Computational thinking”. Commun. ACM, 49(3):33-35.

Zilli, Silvana do Rocio (2004) "A Robótica Educacional no Ensino Fundamental: Perspectivas e Prática", Dissertação de Mestrado, Disponível em: https://repositorio.ufsc.br/bitstream/handle/123456789/86930/224814.pdf?sequence= 1, Acesso em: 15 de junho de 2019. 